OPEN ACCESS

Edited by:

Victor Réville,

UMR5277 Institut de recherche en astrophysique et planétologie (IRAP),

France

Reviewed by: Ivan Vasko,

University of California, Berkeley, United States

Owen Wyn Roberts,

Space Research Institute, Austria

${ }^{*}$ Correspondence: Marian Lazar marian.lazar@kuleuven.be

Specialty section: This article was submitted to

Stellar and Solar Physics,

a section of the journal Frontiers in Astronomy and Space Sciences

Received: 15 September 2021 Accepted: 14 December 2021 Published: 19 January 2022

Citation: Lazar M, López RA, Shaaban SM Poedts S, Yoon PH and Fichtner $H$ (2022) Temperature Anisotropy Instabilities Stimulated by the Solar Wind Suprathermal Populations. Front. Astron. Space Sci. 8:777559. doi: 10.3389/fspas.2021.777559

\section{Temperature Anisotropy Instabilities Stimulated by the Solar Wind Suprathermal Populations}

\author{
Marian Lazar ${ }^{1,2 *}$, R.A. López ${ }^{3}$, Shaaban Mohammed Shaaban ${ }^{4}$, Stefaan Poedts ${ }^{1,5}$, \\ Peter Haesung Yoon ${ }^{6}$ and Horst Fichtner ${ }^{2}$ \\ ${ }^{1}$ Centre for Mathematical Plasma-Astrophysics, KU Leuven, Leuven, Belgium, ${ }^{2}$ Institut für Theoretische Physik IV, Ruhr- \\ Universität Bochum, Bochum, Germany, ${ }^{3}$ Departamento de Física, Universidad de Santiago de Chile, Santiago, Chile, \\ ${ }^{4}$ Theoretical Physics Research Group, Physics Department, Faculty of Science, Mansoura University, Mansoura, Egypt, ${ }^{5}$ Institute \\ of Physics, University of Maria Curie-Skłodowska, Lublin, Poland, ${ }^{6}$ Institute for Physical Science and Technology, University of \\ Maryland, College Park, MD, United States
}

This review paper compiles recent results obtained by the present group of authors describing the effects of suprathermal populations present in space plasmas (up to a few keVs) on temperature anisotropy instabilities. Of particular interest are the electromagnetic cyclotron and firehose excitations, which play a major role in limiting temperature anisotropy, resulting, for instance, from the adiabatic expansion of the solar wind. Relying on a rigorous modeling and interpretation of the observed velocity distributions, both theoretical models and numerical simulations indicate a systematic stimulation of these excitations in the presence of suprathermal populations of electrons or protons. Moreover, the enhanced fluctuations react back on particles, and determine a faster and deeper relaxation of their anisotropy. The present comparative analysis suggests that previous studies, considering only quasithermal low-energy populations, may have significantly underestimated these excitations and their implications in various applications in space plasmas.

Keywords: instabilities, solar wind, temperature, suprathermal particles, particle velocity distribution

\section{INTRODUCTION}

With the first in-situ explorations of solar wind plasmas in interplanetary space, the kinetic (microand mesoscopic) properties of plasma populations became of great interest (Olbert et al., 1968; Vasyliunas, 1968; Gary, 1993). These properties are mainly revealed by the fluxes and velocity distributions of particles measured in-situ, which suggest that, locally, particle plasma populations (electrons, protons, or minor ions) are not in thermal equilibrium. The non-equilibrium features frequently reported by the observations are kinetic anisotropies, such as temperature anisotropy or beam populations (Marsch, 2006), phase space density gradients (Page et al., 2021), as well as suprathermal populations (e.g., halo, beaming populations), with energies up to a few keVs (Olbert et al., 1968; Vasyliunas, 1968; Collier et al., 1996; Maksimovic et al., 2005; Štverák et al., 2008; Mason and Gloeckler, 2012; Tong et al., 2019a). By comparison to the low-energy, or core populations (up to a few tens of eV), which are well reproduced by the standard Maxwellian models, suprathermal populations are less dense (but hotter), and require a more laborious approach based on the Kappa ( $\kappa$-power-law) distributions (Pierrard and Lazar, 2010; Viñas et al., 2015; López et al., 2021). These are probably the main reasons why suprathermal populations have been ignored for decades in the analysis of anisotropic temperatures and related wave excitations, usually invoking the dominance of the core population and the simplicity of standard Maxwellian models, see Ref. (Gary, 1993; Yoon, 
2017). and references therein. However, suprathermal populations (e.g., electron halo) are much hotter and more anisotropic than quasi-thermal core (Maksimovic et al., 2000; Štverák et al., 2008; Pierrard et al., 2016; Lazar et al., 2020), suggesting a significant implication of suprathermals in the kinetic excitations of wave fluctuations and instabilities. Moreover, in recent years important progresses have been made, both theoretically and numerically, by developing kinetic approaches relying on the anisotropic Kappa distribution functions. These models become thus able to describe the observed suprathermal populations (Pierrard and Lazar, 2010; Lazar et al., 2012), and also their contributions to kinetic effects and instabilities of plasma particles (Shaaban et al., 2019a; Lazar et al., 2019; López et al., 2019; López et al., 2021).

In the present review we compile recent results consistently and rigorously describing the effects of suprathermal populations on electromagnetic (EM) excitations induced by the temperature anisotropy of major plasma species in solar wind plasma, i.e., electrons and protons. The present review is largely confined to the works carried out by the present authors, and is not meant to cover the broader aspects regarding the fundamental properties and origins of suprathermal charged particle distributions. Particularly interesting are the EM cyclotron and firehose excitations, known as plasma microinstabilities, or kinetic instabilities, which enhance the smallscale magnetic fluctuations observed in the solar wind (Bale et al., 2009; He et al., 2011; Wilson et al., 2013; Lion et al., 2016; Wicks et al., 2016; Roberts et al., 2017). These instabilities and the resulting enhanced fluctuations should play a major role in selfregulating the properties of solar wind plasma, especially in the absence of particle-particle collisions whose frequency decreases significantly with increasing heliocentric distance (Štverák et al., 2008; Bale et al., 2009; Klein et al., 2018). Also, compared to lowenergy (core) populations, suprathermal particles are less affected by collisions (Maksimovic et al., 2005), but should be more susceptible to kinetic instabilities. Moreover, the free energy of suprathermal particles is expected to stimulate kinetic instabilities, and increase the level of magnetic fluctuations. Despite these expectations, a large number of dispersion and stability analyses predict the opposite, especially an inhibition of these instabilities, for many conditions specific to the solar wind (Lazar and Poedts, 2009; Mace and Sydora, 2010; Lazar et al., 2011; Lazar et al., 2013). Such contradictory outcomes led to further investigations (Lazar et al., 2015; Lazar et al., 2016), which showed that these studies did not use the original Kappa distribution, but another simplified form assuming the corresponding kinetic temperature (as given by the second order moment) independent of the power exponent $\kappa$ (Hau et al., 2009; Hellberg et al., 2009; Livadiotis and McComas, 2012). Empirically introduced by Olbert (Olbert et al., 1968) and Vasyliunas (Vasyliunas, 1968), the original Kappa model (see also section 2 below) enables realistic interpretation of suprathermal populations, and produces reliable results (Lazar et al., 2015), which show rather a stimulation of the temperature anisotropy instabilities and an enhancement of the resulting EM fluctuations in the presence of suprathermal populations (Thorne and Summers, 1991; Lazar et al., 2015;
Lazar et al., 2016; Shaaban et al., 2019a; Lazar et al., 2019; López et al., 2019).

Section 2 presents the essence of such a realistic interpretation of suprathermal populations, which enhance the high-energy tails of the anisotropic bi-Kappa distribution, and are highlighted by contrast with the bi-Maxwellian (quasi-thermal) core. In Sections 3 and $\mathbf{4}$ we discuss the most important instabilities triggered by the temperature anisotropy of electrons and protons, the dominant species in the solar wind. Selected are the results from advanced quasi-linear (QL) theories and from numerical simulations, those able to show the extended evolution of the excited fluctuations, as well as their action back on the particles leading to the relaxation of their temperature anisotropy. The last section (Section 5) brings together the main conclusions and a series of perspectives of the present review study.

\section{MODELING SUPRATHERMAL POPULATIONS}

The modeling of suprathermal populations and their implications is often based on the Kappa distribution functions, introduced to reproduce the observed electron distributions with energies of up to a few eVs (Olbert et al., 1968; Vasyliunas, 1968). In such a case, the Kappa power-law is applied as a global (fitting) model, that incorporates both the low-energy electron core and the suprathermal population enhancing the high-energy tails of the observed distribution. Not only electrons, but also protons and minor ions observed in the solar wind exhibit such velocity distributions (Collier et al., 1996; Mason and Gloeckler, 2012).

The anisotropic model used to describe populations with anisotropic temperatures (i.e., $A=T_{\perp} / T_{\|} \neq 1$ ) is the bi-Kappa distribution (Summers and Thorne, 1991)

$$
f_{\kappa}\left(v_{\perp}, v_{\|}\right)=\frac{1}{\pi^{3 / 2} \theta_{\perp}^{2} \theta_{\|}} \frac{\Gamma(\kappa+1)}{\kappa^{3 / 2} \Gamma(\kappa-1 / 2)}\left[1+\frac{v_{\perp}^{2}}{\kappa \theta_{\perp}^{2}}+\frac{v_{\|}^{2}}{\kappa \theta_{\|}^{2}}\right]^{-\kappa-1},
$$

normalized to unity $\int d^{3} v f_{\kappa}=1$, and written in terms of the normalization velocity parameters $\theta_{\|, \perp}$ (where $\|, \perp$ indicate the components defining the anisotropy with respect to the uniform magnetic field lines). From the second-order moments of Kappa distribution we find the velocity parameters directly related to the corresponding components of the (Kappa) kinetic temperature

$$
T_{\kappa, \|, \perp}=\frac{\kappa}{\kappa-3 / 2} \frac{m \theta_{\|, \perp}^{2}}{2 k_{B}},
$$

for particle populations of mass $m$ (and $k_{B}$ is the Boltzmann constant). These kinetic temperatures are positively defined only for a power exponent $\kappa>3 / 2$.

Widely applied theoretically (Lazar and Poedts, 2009; Mace and Sydora, 2010; Lazar et al., 2011; Lazar et al., 2013; Shaaban et al., 2019a; Lazar et al., 2019; López et al., 2019), the bi-Kappa model has also been used in more recent and refined observations, as a partial fit reproducing only the suprathermal components of the observed distributions, e.g., halo or strahl populations, while the low-energy core was described by a more standard bi-Maxwellian distribution (Maksimovic et al., 2005; Štverák et al., 2008; Tong 
et al., 2019a; Wilson III et al., 2019; Scherer et al., 2020). Such a dual or even multi-component description using Kappa models may offer valuable insights distinguishing between various components, e.g., core, halo and, eventually, beaming (or strahl) populations, their properties and origin. In such a case the theory becomes not only complicated, due to the multitude of parameters involved, but also limited to the specific case defined by the set of parameters considered in the approach (Lazar et al., 2014; Shaaban et al., 2016; Lazar et al., 2018a; Lazar et al., 2018b; Shaaban et al., 2019b).

For plasma instabilities driven by the temperature anisotropy of the observed (gyrotropic) distributions relevant are the most unstable cases combining both the core and suprathermal halo populations with similar anisotropies, either both with $A=T_{\perp} / T_{\|}$ $>1$, or both with $A=T_{\perp} / T_{\|}<1$ (Lazar et al., 2018a; Lazar et al., 2018b; Shaaban et al., 2019b). For instance, the electron plasma states with a dual core-halo composition, showing reduced relative drifts but similar (correlated-like) anisotropies, are often revealed by the solar wind observations (Štverák et al., 2008; Pierrard et al., 2016; Wilson III et al., 2019), and can be described by a global (bi-)Kappa (Lazar et al., 2017a), as also suggested in the early theories and observations (Pierrard and Lazar, 2010) ${ }^{1}$. Such a simplified approach cannot provide details about the components like core, halo or additional beams, but has a particular advantage in the analysis of suprathermal halo populations, facilitating the understanding of their implications in the excitation of anisotropic temperature instabilities. Thus, the suprathermals can be emphasized and even quantified based on a comparison between the observed (global) Kappa distribution with enhanced high-energy tails (red line in Figure 1) and the Maxwellian low-energy core in the absence of suprathermal population (e.g., dashed blue line in Figure 1) (Lazar et al., 2015; Lazar et al., 2016).

Such a contrasting analysis becomes straightforward, since the quasi-thermal core (subscript $c$ ) is reproduced with a good approximation by the Maxwellian limit (of lower temperature) of the Kappa distribution, i.e., $f_{\kappa \rightarrow \infty}\left(v_{\perp}, v_{\|}\right)=f_{M}\left(v_{\perp}, v_{\|}\right)$(see also Figure 1) (Lazar et al., 2015; Lazar et al., 2016), which reads

$$
f_{c}\left(v_{\perp}, v_{\|}\right) \simeq f_{M}\left(v_{\perp}, v_{\|}\right)=\frac{1}{\pi^{3 / 2} \theta_{\perp}^{2} \theta_{\|}} \exp \left(-\frac{v_{\perp}^{2}}{\theta_{\perp}^{2}}-\frac{v_{\|}^{2}}{\theta_{\|}^{2}}\right) \text {. }
$$

Here the normalization parameters $\theta_{\perp, \|}$ are thermal velocities related to the corresponding components of the core temperature

$$
T_{c, \|, \perp} \simeq \frac{m \theta_{\|, \perp}^{2}}{2 k_{B}}<T_{\kappa, \|, \perp} .
$$

This implies an (approximate) relationship between temperatures (Lazar et al., 2015)

\footnotetext{
${ }^{1}$ Focusing on temperature anisotropy instabilities, in a zero order approximation we can neglect the influence of the relative drift that may exist between core and halo populations. Away from the energetic events or interplanetary shocks this drift is insignificant (much lower than thermal spreads and relative drifts of beam-strahl populations) (Wilson III et al., 2019), being not even captured by the observations (Štverák et al., 2008)
}

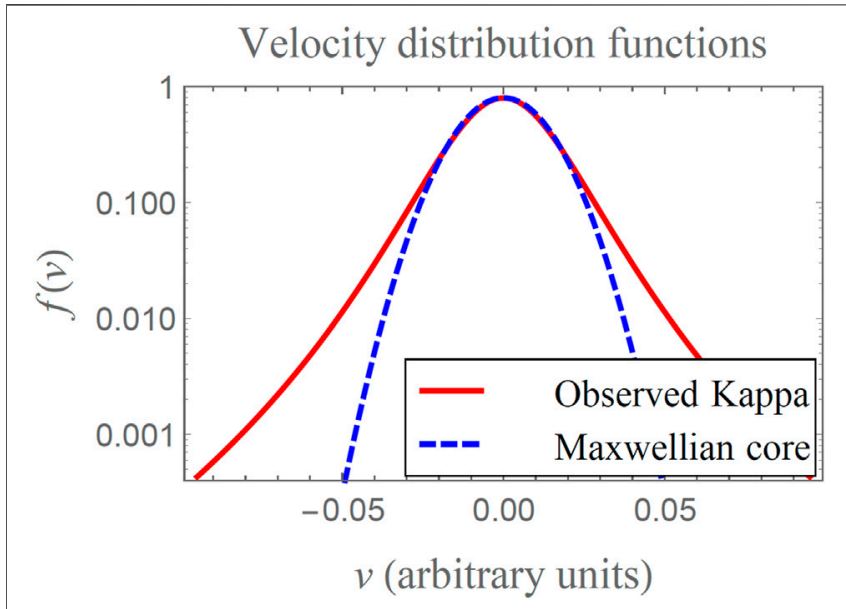

FIGURE 1 | 1D representation contrasting the observed (global) Kappa distribution with enhanced high-energy tails (red line) with the Maxwellian lowenergy core (dashed blue line) in the absence of suprathermal population.

$$
T_{\kappa, \|, \perp} \simeq \frac{\kappa}{\kappa-3 / 2} T_{c, \|, \perp}>T_{c, \|, \perp}
$$

and between the corresponding plasma beta parameters, for instance, the parallel components

$$
\beta_{\kappa, \|} \simeq \frac{\kappa}{\kappa-3 / 2} \beta_{c, \|}>\beta_{c, \|},
$$

commonly invoked in the dispersion analysis. The plasma beta is defined (kinetically) as $\beta=8 \pi n k_{B} T / B_{0}^{2}$, where $n$ is the number density of plasma particles of temperature $T$, and $B_{0}$ is the strength of the uniform magnetic field. In the analysis below, we will specify the values for the plasma beta corresponding to the (bi-)Maxwellian limit, i.e., $\beta_{c, \|}=\beta_{\|}$, and for the power exponent $\kappa$. It becomes also clear that this bi-Maxwellian limit considerably facilitates the comparison with the bi-Kappa distribution, both in analytical and numerical computations, providing results of a general validity (Lazar et al., 2015; Lazar et al., 2016; Shaaban et al., 2021b). In this case one can conclude on the implications of suprathermal populations without resorting to a laborious statistical study of the results obtained for each core-halo combination.

If we reiterate those studies which invoke another comparison of the Kappa distribution with a Maxwellian limit of the same (kinetic) temperature (Hellberg et al., 2009; Livadiotis and McComas, 2012), i.e., $T_{M}=T_{\kappa}$, their results do not have the same relevance, and cannot highlight suprathermal populations and their implications (Hau et al., 2009; Lazar et al., 2015; Lazar et al., 2016) (a graphical comparison of a bi-Kappa with both bi-Maxwellian limits can be found in (Lazar et al., 2016)). Many of the early analyses of temperature anisotropy instabilities were also affected by such a simplified approach (Lazar and Poedts, 2009; Mace and Sydora, 2010; Lazar et al., 2011). However, more recent studies based on a realistic contrast between a (bi-)Kappa with its (bi-)Maxwellian core, as illustrated in Figure 1, have reached concurring conclusions providing a consistent interpretation of the suprathermal 

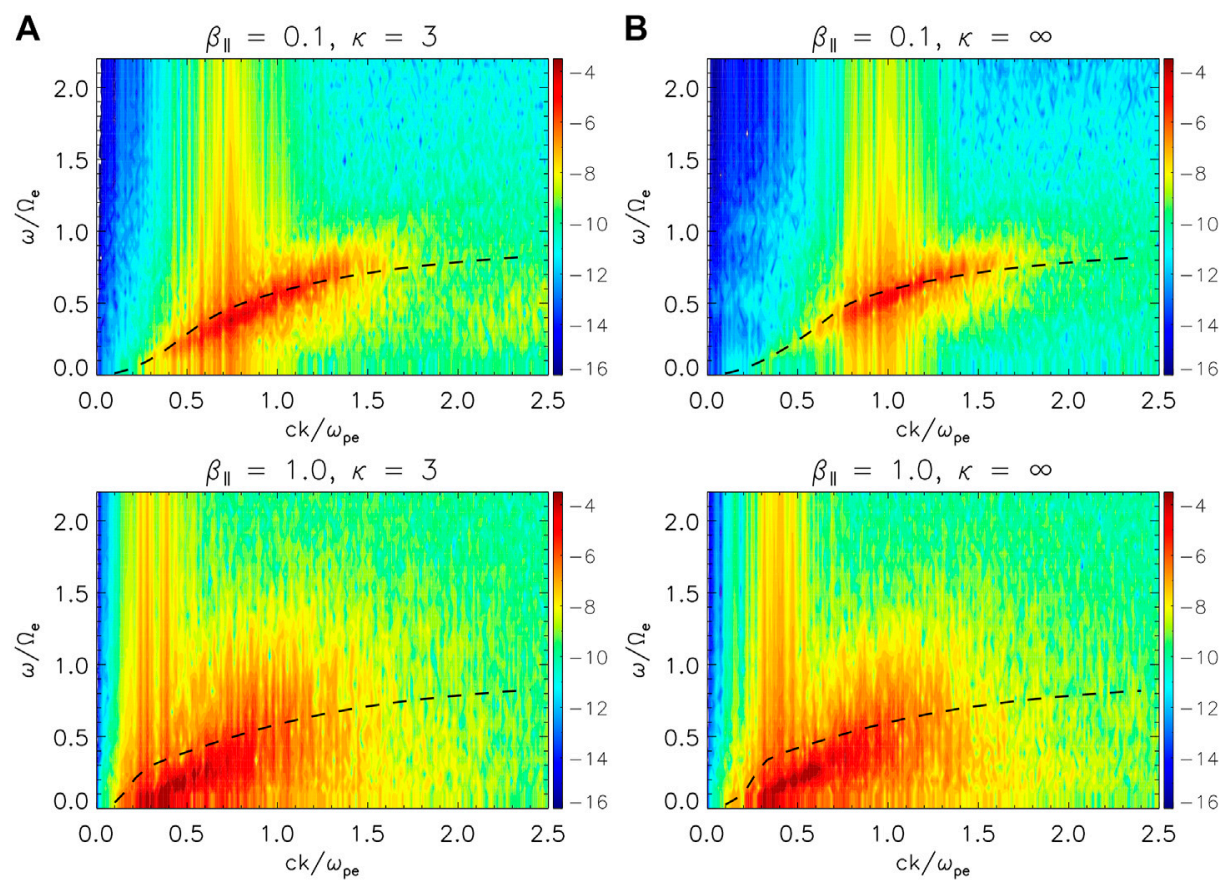

FIGURE 2 | Whistler fluctuations computed from PIC simulations for bi-Kappa (A) and for bi-Maxwellian (B) electrons with $A_{e}(0)=4.0, \beta_{e}, \|(0)=0.1$ (top) and $\beta_{\mathrm{e}, \|}(0)=1.0$ (bottom): peaks of the wave magnetic power (color coded) align to the linear dispersion in the frequency $(\omega)-$ wave-number $(k)$ space.

populations and their contribution to these instabilities (Lazar et al., 2016; Shaaban et al., 2019a; López et al., 2019). In the following we will revisit these more reliable studies, paying particular attention to the results obtained with advanced QL theories and numerical simulations.

\section{INSTABILITIES DRIVEN BY ANISOTROPIC ELECTRONS}

\subsection{Whistler Instability $\left(A_{e}>1\right)$}

The enhanced magnetic fluctuations in the range of whistler waves have been reported in space plasmas since the ' $80 \mathrm{~s}$, especially in association with interplanetary shocks and corotating interaction regions (CIRs) (Coroniti et al., 1982; Lin et al., 1998; Wilson et al., 2013). Over the past decade, highperformance detectors were also able to measure these fluctuations in more quiet environments, such as the pristine solar wind, showing that the occurrence of whistler waves is related not only to electron suprathermal (halo, strahl) populations carrying the heat-flux, but also to the electron temperature anisotropy (Vinas et al., 2010; Lacombe et al., 2014; Stansby et al., 2016; Tong et al., 2019b) ${ }^{2}$. So far, a link between whistler fluctuations and the anisotropic temperature of

${ }^{2}$ Observationally, there can be shortcomings in using total moments of the velocity distributions measured in-situ, and for a rigorous interpretation one may need more sophisticated analyses to differentiate between electron populations and quantify their properties (Wilson III et al., 2019; Vinas et al., 2010). electrons (subscript $e$ here in the next), i.e., $A_{e}=T_{e, \perp} / T_{e, \|}>1$, has been established rather indirectly. Thus, the threshold of the whistler instability has been found to shape the limits of this anisotropy measured in-situ (Štverák et al., 2008), providing another strong evidence on the role self-generated instabilities can play in determining the solar wind properties.

In order to outline the effects of suprathermal electrons on the whistler instability, here we revisit and refine the more or less recent analyses of this instability in the solar wind conditions (Lazar et al., 2013; Lazar et al., 2015; Lazar et al., 2019), focusing on the results from $1 \mathrm{D}$ particle-in-cell (PIC) simulations. We chose set-ups similar to those in Ref. (Lazar et al., 2019), and with outcomes in very good agreement with linear and QL theory. Thus, in Figure 2 we show the magnetic field fluctuations obtained from PIC simulations (as $\left|\mathrm{FFT}_{(x, t)}\left(B_{y}-i B_{z}\right)\right|^{2}$ in the color bar, where the fast Fourier transform is taken along the spatial and temporal dimensions, see also Ref. (Lazar et al., 2019)), for two sets of parameters $A_{e}(0)=4.0, \beta_{\|}(0)=0.1$ (top) and $\beta_{\|}(0)=1.0$ (bottom). The whistler nature of these fluctuations is confirmed by their alignment along the dispersion curves of whistler modes, i.e., the wave frequency (normalized to the electron gyro-frequency, $\Omega_{e}=|e| B_{0} / m_{e} c$ ) derived from linear theory as a function of the wave number (normalized by the inverse of electron skin depth $\omega_{p e} / c$, where $\omega_{p e}=\left(4 \pi n e^{2} / m_{e}\right)^{1 / 2}$ is the plasma frequency and $c$ is the speed of light in vacuum). Comparison between the bi-Kappa (left) and bi-Maxwellian (right) electrons shows an enhancement of the wave power in the presence of suprathermal electrons (left panels). As expected, this difference giving us the contribution of suprathermals is reduced when electrons have (initially) a higher kinetic energy or 


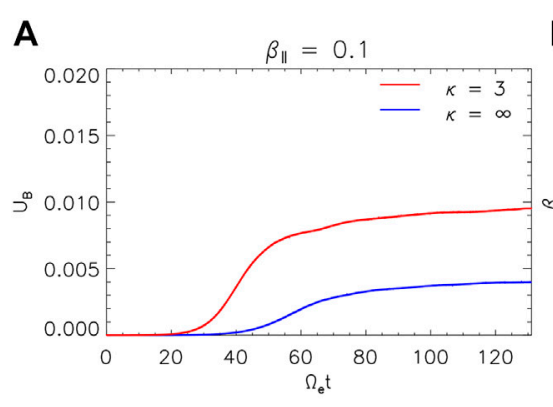

$\beta_{\|}=1.0$

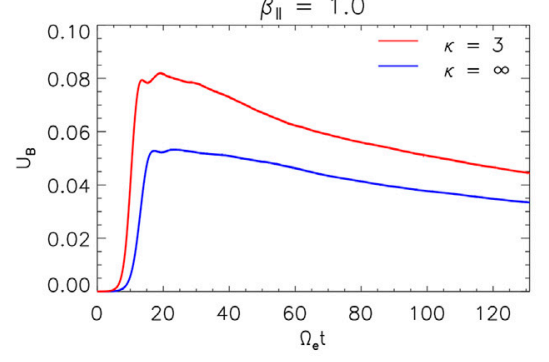

B

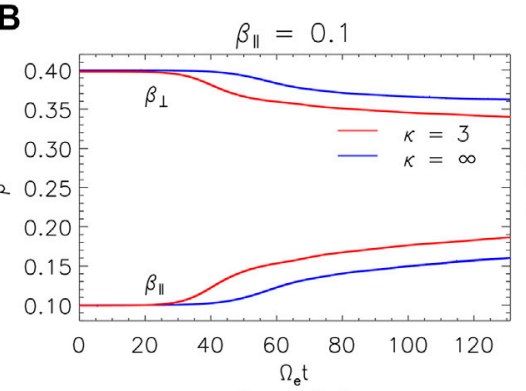

$\beta_{\|}=1.0$

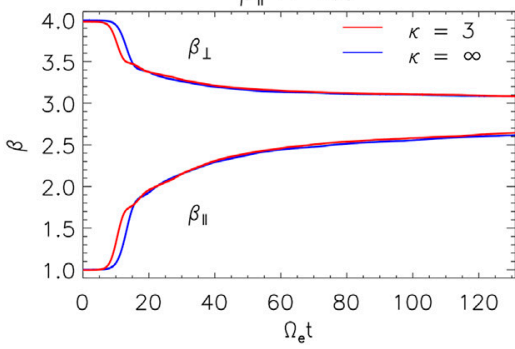

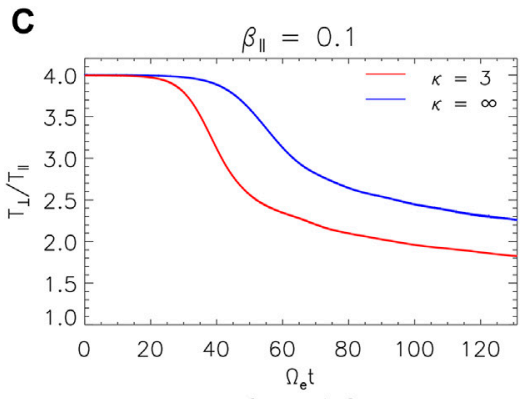

$\beta_{\|}=1.0$

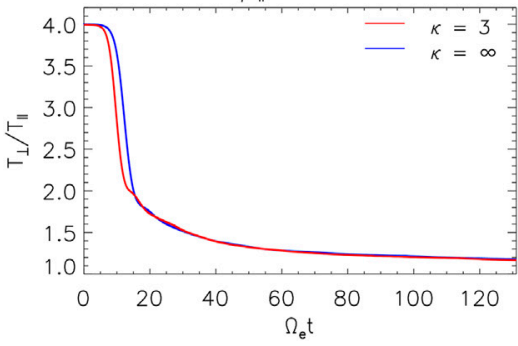

FIGURE 3 | Temporal variation of whistler instability from PIC simulations for bi-Kappa (red) and bi-Maxwellian electrons (blue): magnetic field power (A), plasma betas (B) and temperature anisotropy (C). Initial parameters are $A_{e}(0)=4.0, \beta_{\mathrm{e}, \|}(0)=0.1$ (top) and $\beta_{\mathrm{e}, \|}(0)=1.0$ (bottom).

temperature, reflected by a higher beta parameter, i.e., $\beta_{e, \|}(t=0)=$ 1.0 (bottom panels).

Figure 3 displays temporal variations of the magnetic energy density (normalized to the electron density at rest) $U_{B}(t)=(1 /$ $(8 \pi)) \int d x \delta B^{2}(x, t)$ (left), temperature anisotropy (middle) and plasma betas (right), for an initial $\beta_{e, \|}(t=0)=0.1$ (top), and $\beta_{e, \|}(t=0)=1.0$ (bottom). The results are obtained as function of the normalized time $\tau=\Omega_{e} t$. In the presence of suprathermals electrons (red lines for $\kappa=3$ ) all cases show a stimulation of the unstable fluctuations, enhancing the magnetic wave power and deepening the relaxation of the anisotropy. In the first case, for a low plasma beta, the maximum wave power reached at the saturation is more than two times higher in the presence of suprathermals. When electrons have a higher (initial) temperature or higher beta parameter, i.e., $\beta_{e, \|}(t=0)=1.0$, the instability is triggered earlier in time, but the effects of suprathermals are weaker. In this case, the wave power reached at the saturation is enhanced only 1.5 times and the influence on the relaxation of the anisotropy is also significantly reduced.

However, in the second run for electrons with higher betas, i.e., $\beta_{e, \|}(t=0)=1.0$ (bottom panels), the amplitude of whistler fluctuations is much higher, with a magnetic power reached at saturation (bottom-left panel) almost one order of magnitude higher than first case (top-left panel). This explains the deeper relaxation of the anisotropy (middle and right panels), and may also help us to understand why the fluctuating magnetic power computed in Figure 2 deviates from the linear dispersion relation, and why this deviation increases with $\beta_{e, \|}(t=0)$. These fluctuations, coded with colors in Figure 2, represent the total magnetic field power, integrated over the whole interval of time simulation, and because the temperature components and anisotropy change during the run, as shown in Figure 3, the corresponding dispersion of the whistler fluctuations also change, thus explaining the deviations, more or less significant, obtained in Figure 2. These deviations also seem to depend on the noise level in the simulations, both increasing with plasma beta, as argued in Ref. (Lazar et al., 2019). from a comparison of the results obtained for $\beta_{e, \|}(t=0)=0.1$, and $\beta_{e, \|}(t=0)=2.0$.

Temporal variations from Figure 3 compare very well with those obtained from a QL approach, not shown here, but provided in Ref. (Lazar et al., 2019), showing that the level of whistler fluctuations driven by a moderate electron temperature anisotropy, similar to the solar wind observations, does not reach very high amplitudes. Associated with the anisotropy of electrons, i.e., anisotropic temperature, heat flux, whistler wave fluctuations measured in the solar wind confirm such low level of amplitudes (Wilson et al., 2013; Tong et al., 2019b). Therefore, highly nonlinear effects, like non-linear wave-wave interaction (e.g., interaction of three-waves, non-linear wave damping) or particle trapping may not involve, and the QL saturation is only the result of the scattering of electrons by enhanced fluctuations, which leads to the relaxation of their temperature anisotropy.

\subsection{Electron Firehose Instabilities $\left(A_{e}<1\right)$}

Firehose instabilities are excited by an anisotropy (excess) of temperature in direction parallel to the magnetic field, i.e., $A_{e}=$ $T_{e, \perp} / T_{e, \|}<1$. We first discuss the electron firehose $(\mathrm{EFH})$ instabilities, which have two distinct branches of unstable modes. Thus, the finite frequency firehose modes $(\omega \neq 0$, periodic) may propagate along the magnetic field and at small angles, while the aperiodic firehose mode $(\omega=0)$, propagates only in oblique directions, and develops more rapidly with 

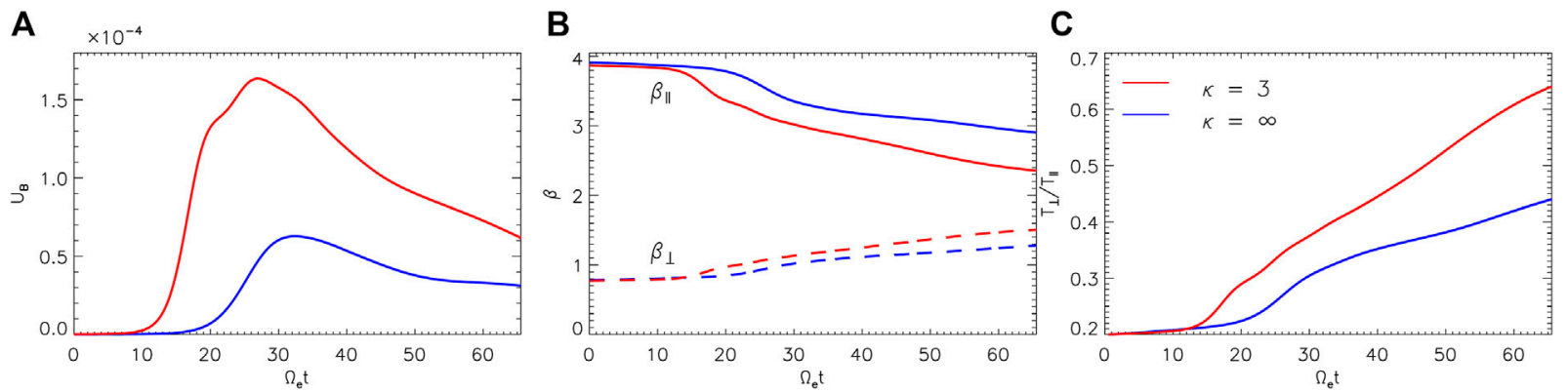

FIGURE 4| The results from PIC simulations of the O-EFH instability for bi-Kappa (red) and bi-Maxwellian (blue) electrons: temporal evolution for the magnetic wave energy (A), proton plasma beta components (B), and the relaxation of temperature anisotropy (C).

A

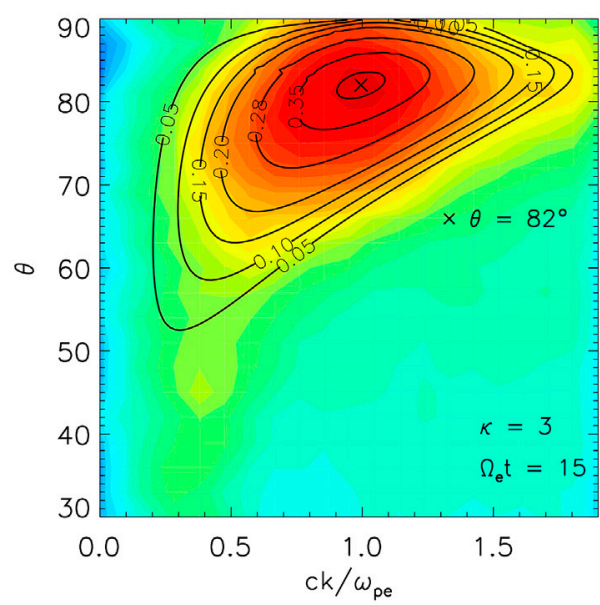

B

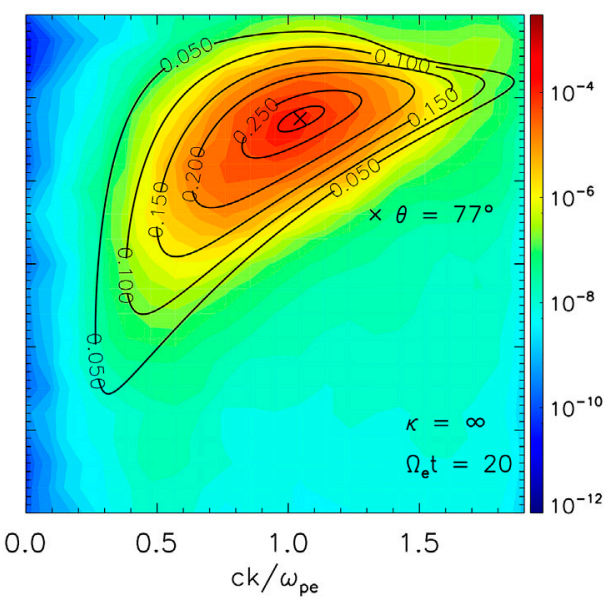

FIGURE 5|Power spectra (color coded) of O-EFH fluctuations $\left(\delta B_{z}\right.$ component) from PIC simulations, for bi-Kappa protons with $\kappa_{e}=3$ (A) and bi-Maxwellian limit $\kappa \rightarrow \infty$ (B). Contours of the linear growth rates $\left(\gamma / \Omega_{e}\right)$ show a very good agreement with the simulations.

(maximum) growth rates much higher than the periodic mode ( $\mathrm{Li}$ and Habbal, 2000; Camporeale and Burgess, 2008; Shaaban et al., 2019a); see, for instance, Figure 4, top panel, in Ref. (Shaaban et al., 2019a). Because of this, only the oblique EFH (O-EFH) instability is revisited here, based on a retrospective of linear approach for bi-Kappa distributed electrons (Shaaban et al., 2019a), but also on the results from long-term runs of this instability in 2D PIC simulations (López et al., 2019).

In Figure 4 we show temporal evolution from 2D PIC simulations for the magnetic energy density (left), electron plasma beta components (middle), and the relaxation of temperature anisotropy (right). These results are obtained for an initial anisotropy $A_{e}(t=0)=0.2$ and plasma beta $\beta_{e, \|}(t=0)$ $=4$ (with the same normalized time $\tau=\Omega_{e} t$ ), and reproduce those from Ref. (López et al., 2019). In order to outline the effects of suprathermals we compare the evolution obtained for the bi-Kappa electrons ( $\kappa=3$, red lines) with that for bi-Maxwellian limit $(\kappa=\infty$, blue line). These temporal profiles show a significant stimulation of the instability, with the magnetic wave energy of the O-EFH fluctuations enhanced in the presence of suprathermal electrons, which in turn leads to a deeper relaxation of the initial temperature anisotropy. However, the resulting firehose fluctuations remain at low levels (much lower than whistler fluctuations above), which may explain the difficulty in detecting them directly in-situ.

Figure 5 combines very suggestively the growth rates (isocontours) predicted by the linear theory for the O-EFH instability, with the fluctuation power spectra (color coded) obtained from PIC simulations. These results are displayed here depending on the propagation angle $\theta$ and the (total) wave number $\kappa$, and show a very good qualitative agreement. Comparison with the bi-Maxwellian limit (right panel) reconfirms the enhancement of firehose fluctuations in the presence of suprathermals (left panel). Also, the maximum (peaking) growth rate moves to slightly higher oblique angles and lower wave-numbers. 

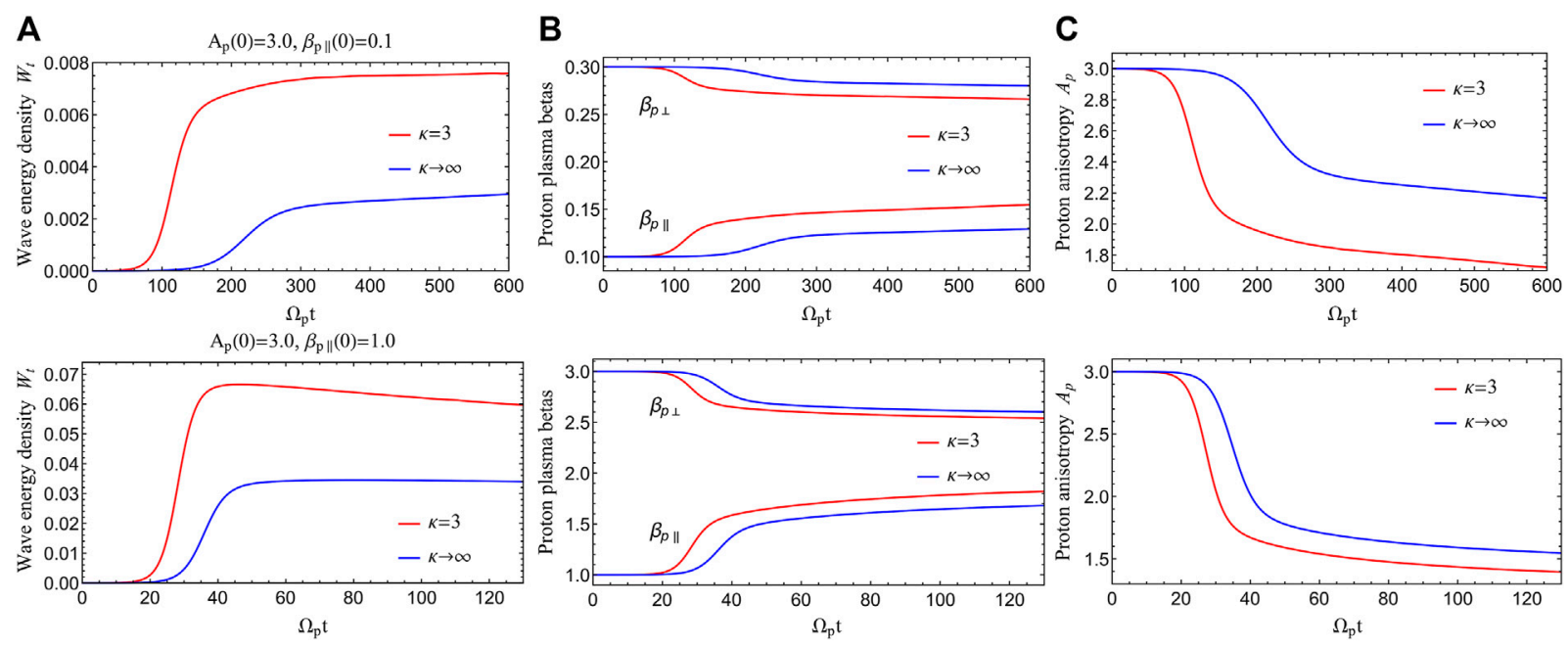

FIGURE 6 | QL evolution of the EMIC instability for bi-Kappa (red) and bi-Maxwellian protons: magnetic energy (A), components of proton plasma beta (B) and the subsequent relaxation of the anisotropy $(\mathbf{C})$, obtained for $A_{p}(t=0)=3.0$ and $\beta_{p\|\|}(t=0)=0.1$ (top) and $\beta_{p \|}(t=0)=1.0$ (bottom).

\section{INSTABILITIES DRIVEN BY ANISOTROPIC PROTONS}

\subsection{EMIC Instability $\left(A_{p}>1\right)$}

The electromagnetic ion-cyclotron (EMIC) modes with frequencies below the proton (subscript $p$ here in the next) gyro-frequency $\Omega_{p}=|e| B_{0} / m_{p} c$ are destabilized by the anisotropic protons with temperature anisotropy $A_{p}=T_{p, \perp} / T_{p, \|}$ $>1$ (Gary, 1993). Enhanced EMIC fluctuations are constantly detected in the solar wind, at various heliographic coordinates (Jian et al., 2009; Podesta and Gary, 2011; Wicks et al., 2016; Klein et al., 2018), but also in the Earth's magnetosphere (Anderson et al., 1991; Nguyen et al., 2007), motivating the interest for understanding not only their origin, but also their implications, and, especially, in the presence of suprathermal protons (Lazar, 2012; Yoon and Seough, 2012; Shaaban et al., 2016; Shaaban et al., 2021a). Recently, significant progress has been made by elaborating a new QL theoretical formalism, see Ref. (Shaaban et al., 2021a), capable of describing these effects of suprathermal protons, by using, for the first time, a relevant Kappa approach, such as that described in Section 2 above. Here we revisit and test the results from Ref. (Shaaban et al., 2021a), using the same QL theory and numerical solvers to obtain unstable EMIC solutions for different initial conditions, but still specific to space plasmas.

Figure 6 displays the same temporal variations as in Figure 3, this time for the total (normalized) wave energy density $W_{t}=$ $\int d \tilde{k} \delta W(\tilde{k})$ of the EMIC fluctuations (left), and proton parameters, namely, the plasma beta components (middle) and temperature anisotropy (right). These results are obtained from a QL approach (Shaaban et al., 2021a) for bi-Kappa (red) and biMaxwellian (blue) protons with an initial anisotropy $A_{p}(t=0)=$ 3.0, and two distinct cases for low $\beta_{p, \|}=0.1$ (top) and high $\beta_{p, \|}=$ 1.0 (bottom). All these temporal profiles resemble those corresponding to Figure $\mathbf{1}$ for whistlers, which are destabilized by the same cyclotron resonance, but with electrons. For a low (initial) $\beta_{p, \|}=0.1$ (top panels) the resulting fluctuations reach low amplitudes (even at the saturation), and does not contribute much to the relaxation of anisotropy. Suprathermal protons have the same stimulating effect on the EMIC fluctuations, which in turn determine larger variations of the plasma beta components and a deeper relaxation of the anisotropy. In the second case (bottom panels), plasma beta is one order of magnitude higher, and the wave energy density reached at the saturation is also markedly increased, contributing also to a deeper relaxation of the anisotropy. The effect of suprathermals is reduced, as reflected not only by the wave energy density, but also the proton parameters.

Corresponding to these two cases, in Figure 7 we show the variation in time of the spectral (normalized) wave energy density $W(\tilde{k})=\delta B^{2}(\tilde{k}) / B_{0}^{2} \quad$ (color coded), from high to low wavenumbers, and even much lower in the presence of suprathermal protons. The significant enhancement of the EMIC fluctuations reached at the saturation is also evident, not only in the presence of suprathermals, but also with the increase of $\beta_{p, \| \cdot}$. The wave-number decreases, especially after saturation, because temperature anisotropy decreases. Accordingly, growth rates also become lower, see linear theory (Shaaban et al., 2021a), but remain high enough to amplify already large amplitude fluctuations (after saturation), and to explain increased levels of $W_{t}(\tilde{k})$.

\subsection{Proton Firehose Instabilities $\left(A_{p}<1\right)$}

If protons exhibit an opposite anisotropy, i.e., $A_{p}=T_{p, \perp} / T_{p, \|}<1$, the theory predicts two other branches of proton firehose (PFH) instabilities, similar to the EFH instabilities (Gary, 1993; Hellinger et al., 2006; Maneva et al., 2016; Hunana and Zank, 2017). However, in this case the periodic $\mathrm{PFH}$ instability, propagating parallel to the magnetic field, can be more competitive, with growth rates exceeding those of the aperiodic (oblique) branch (Gary, 1993; Hellinger et al., 2006; Maneva et al., 2016; Hunana 

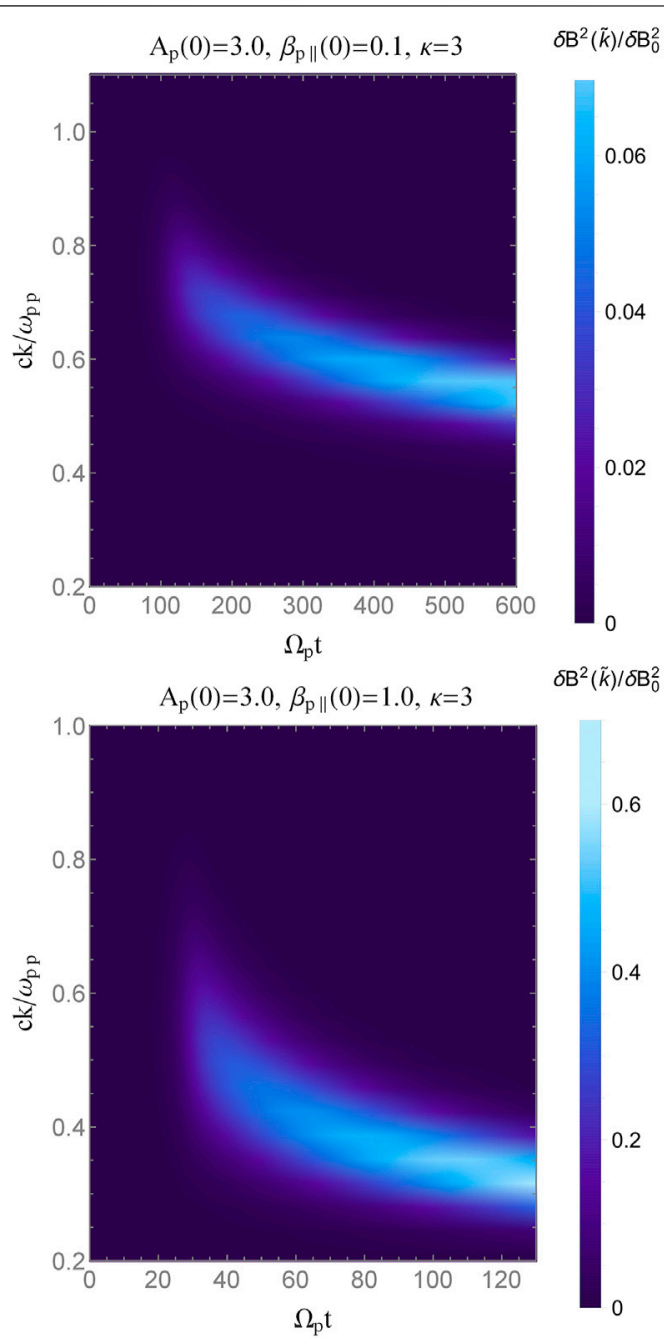
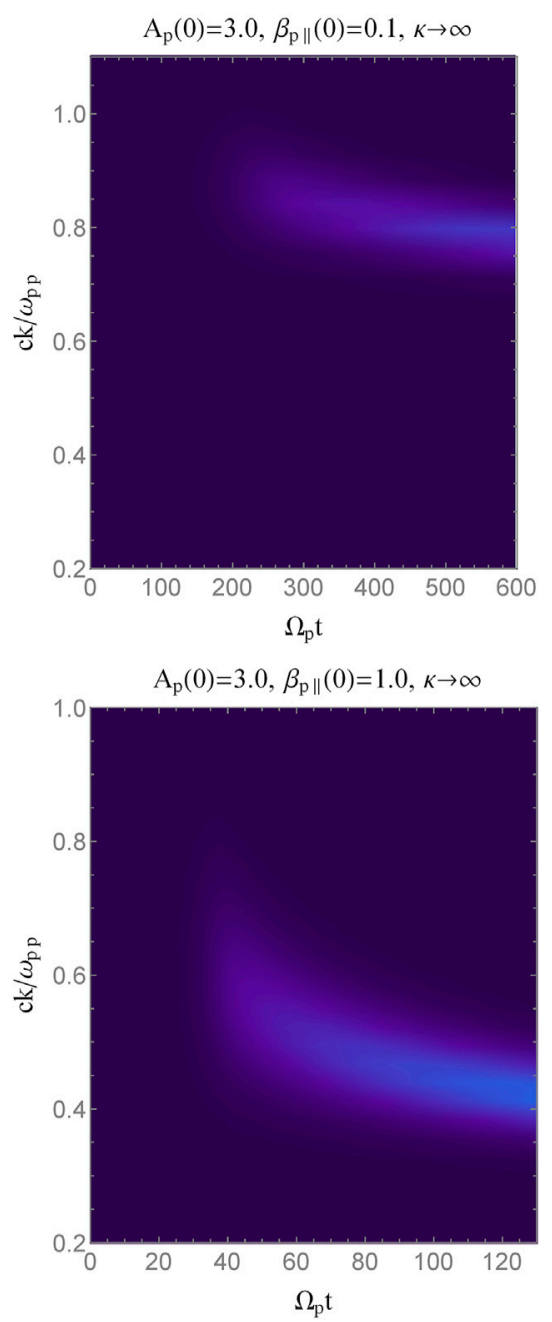

FIGURE 7 | Temporal variation of the wave-number spectra of EMIC magnetic wave energy density for the same cases in Figure $\mathbf{6}$.

and Zank, 2017). Therefore, we will restrict our present analysis only to the P-PFH instability, and focus on the influence of suprathermal protons by using the same relevant comparison described in Section 2. We have used the new QL formalism reported recently in Ref. (Shaaban et al., 2021b), but to derive unstable PFH modes for (slightly) different initial parameters of proton population, thus testing the consistency of previous results.

Figure 8 displays in the same format the most relevant variations in time of the wave energy density (left), components of proton beta parameter (middle), and temperature anisotropy (right). To outline the effects of suprathermal protons, we contrast the results obtained for anisotropic protons with bi-Kappa (red) and bi-Maxwellian (blue) distributions, with initial parameters $A_{p}(t=0)=0.5$ and $\beta_{p \|}(t=0)=3.0$. As also shown in Ref. (Shaaban et al., $2021 \mathrm{~b})$, suprathermals lead to higher wave energy density $\left(W_{t}\right.$, left panel), and the enhanced fluctuations naturally determine stronger variations of the proton plasma beta components (middle panel), and a deeper relaxation of their anisotropy (right panel). However, in this case the instability ignites much faster in the presence of suprathermals, that seems characteristic to lower plasma beta conditions (compare, for instance, cases in Figure 6).

Thresholds of PFH instabilities are often invoked to explain the limits of proton temperature anisotropy (Kasper et al., 2002; Hellinger et al., 2006; Bale et al., 2009; Shaaban et al., 2017), usually at sufficiently large heliocentric distances, where the observations do not confirm a pure adiabatic expansion of the solar wind (Chew et al., 1956; Matteini et al., 2007; Matteini et al., 2012), and particle-particle collisions are too rare to play, alone, the role of an anisotropy constraint (Bale et al., 2009). Enhanced fluctuations observed at $1 \mathrm{AU}$ have been associated with these anisotropies (Bale et al., 2009), although suprathermal proton populations have not been considered yet to provide a complete observational confrontation. In Figure 9 we show thresholds of the $\mathrm{PFH}$ instability, derived in terms of proton parameters, i.e., $A_{p}(<1)$ vs. $\beta_{p \|}$, again, by comparison, for bi-Kappa (red) 

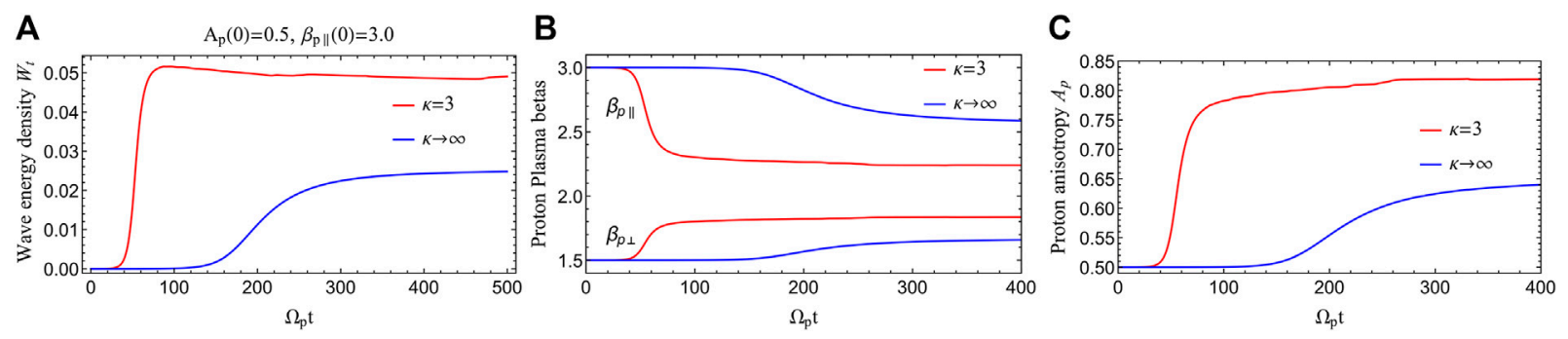

FIGURE 8 | QL evolution of the periodic PFH instability: magnetic energy (A), proton plasma beta (B) and the subsequent relaxation of the anisotropy (C) for anisotropic protons with bi-Kappa (red) or bi-Maxwellian (blue) distributions. Initial parameters are $A_{p}(t=0)=0.5$, and $\beta_{p}, \|(t=0)=3.0$.
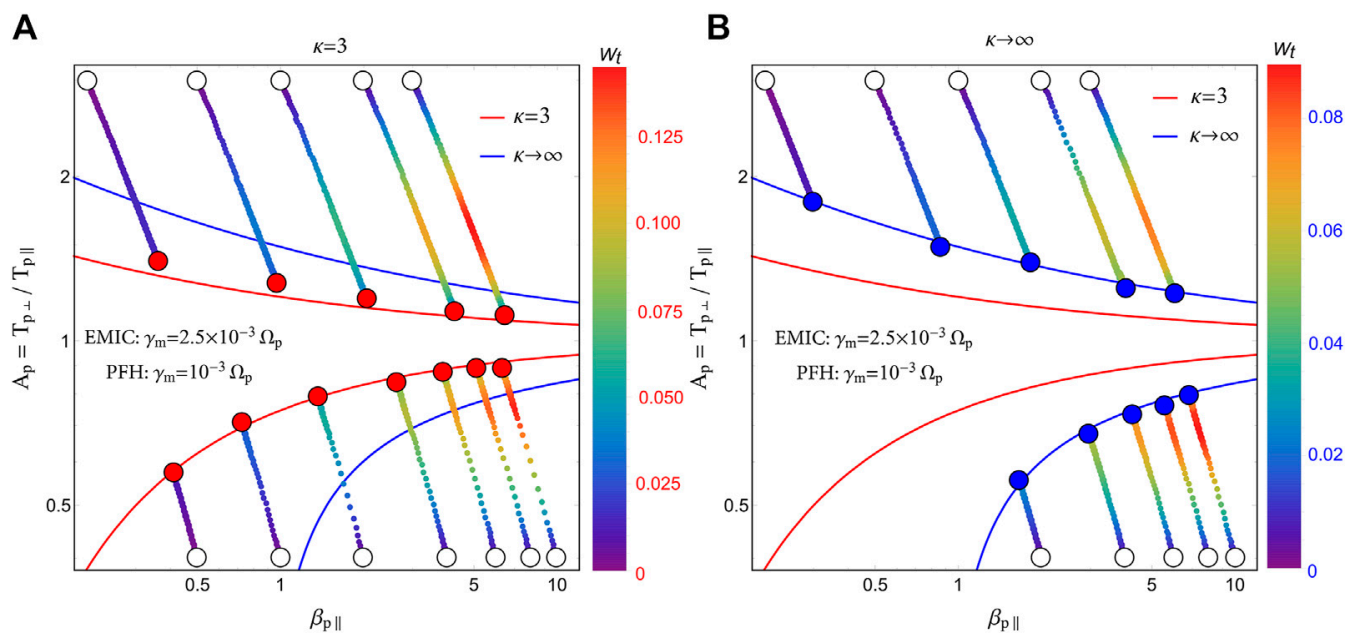

FIGURE 9 |Dynamical paths from QL theory, and the subsequent deeper relaxation of the anisotropy for bi-Kappa electrons (A) comparing to bi-Maxwellian limit (B): the evolution of the anisotropy $\left(A_{p}\right)$ vs. parallel proton beta $\left(\beta_{p, \|)}\right)$ triggered by the EMIC (top) and PFH (bottom) instabilities.

and bi-Maxwellian (blue) protons (Shaaban et al., 2021b). These thresholds are derived from linear theory, see, e.g., in Ref. (Shaaban et al., 2017), and correspond to small maximum growth-rates $\gamma_{m}=10^{-3} \Omega_{p}$ (near the marginal stability, $\gamma_{m}=$ 0 ). Here the stimulating effect of suprathermal protons becomes also evident, causing a significant reduction of the instability threshold. We also display a number of dynamical paths, as obtained from QL theory for bi-Kappa protons in the left panel, and for bi-Maxwellian protons in the right panel (Shaaban et al., 2021b). For each of these runs the instability develops from different initial conditions, i.e., different values of $\beta_{p \|}$, and the final states after saturation align, quite well, along the thresholds derived from the linear theory, providing a supplementary confirmation of these thresholds.

For the sake of completeness, in the same diagrams from Figure 9, but in the upper half (for $A_{p}>1$ ), we also plot the anisotropy thresholds and the QL dynamical paths for EMIC instability (Shaaban et al., 2021a) discussed in Section 4.1. This time thresholds correspond to maximum growth-rates $\gamma_{m}=2.5 \times$ $10^{-3} \Omega_{p}$ (approaching marginal stability, $\gamma_{m}=0$ ), and are derived from linear theory for bi-Kappa (red) and bi-Maxwellian (blue) protons. Their comparison confirms the significant stimulating effect of suprathermal protons on EMIC instability, markedly lowering the anisotropy instability (red lines). The QL dynamical paths for the EMIC instability are also contrasted, for bi-Kappa (left) and bi-Maxwellian (right) protons, and confirm a deeper and more efficient relaxation of the anisotropy in the presence of suprathermal protons. For all cases, the final states after saturation align again along the thresholds predicted by the linear theory. Relaxation of temperature anisotropy $(A \lessgtr 1)$ is a direct consequence of the action of fluctuations generated by instabilities, either EMIC or PFH instabilities. The levels of wave energy density $\left(W_{t}\right)$ of both the EMIC and PFH fluctuations are color coded along dynamical paths, and confirm their enhancement in the presence of suprathermal protons (left panel).

\section{CONCLUSION}

In this paper we have reviewed a number of recent results on the instabilities induced by the temperature anisotropy of plasma particles, under conditions typical for the solar wind and planetary environments, e.g., in Refs. (Lazar et al., 2019; López 
et al., 2019; Shaaban et al., 2021b; Shaaban et al., 2021a). The interest is to outline and show how these instabilities are influenced by suprathermal populations (with energies up to $1 \mathrm{keV}$ ), which are often ignored despite their ubiquity in space plasmas (Gary, 1993; Yoon, 2017). The recent studies we have referred to have the merit of using a rigorous modeling and interpretation of suprathermal populations based on the (anisotropic) Kappa distribution functions, see Section 2 (Lazar et al., 2015; Lazar et al., 2016). What motivated our analysis even more was the fact that all these recent results showed a systematic stimulation of the instabilities induced by temperature anisotropy, whether they are induced by different species (i.e., electrons, protons), or are instabilities of different nature (e.g., cyclotron or firehose) (Lazar et al., 2015; Lazar et al., 2016; Shaaban et al., 2019a; Lazar et al., 2019; López et al., 2019). Therefore, our intention was not only to revisit but also to test these results, as well as theoretical approaches (i.e., linear and QL) and numerical simulations, which were re-applied for the same or slightly modified set-ups, but keeping a parameterization specific to space plasmas.

By formulating these conclusions, we can indeed confirm that suprathermal populations, both electrons and protons, have a systematic stimulating effect not only on cyclotron instabilities, i.e., whistler and EMIC, but also on firehose instabilities, both branches of periodic (or parallel) and aperiodic (or oblique) firehose modes. The unstable fluctuations, quantified by their, e.g., wave power or energy density, can be markedly enhanced by the suprathermal populations, especially when the low-energy (core) populations are initially in low plasma beta conditions. Indeed, comparisons between low and high beta conditions, clearly show that suprathermal populations contribute with an additional kinetic (free) energy, producing similar results as those obtained by increasing the plasma beta parameter. From now on, in-situ measurements can explain the increased levels of these specific fluctuations not only by association with temperature anisotropy (Bale et al., 2009) but also by the presence of suprathermal populations. On the other hand, the resulting enhanced fluctuations should act back on the anisotropic particles, and the results from long runs indicate stronger variations of their parameters in the presence of suprathermals, also leading to a deeper relaxation of temperature anisotropy.

The same stimulating effect is also reflected by the anisotropy thresholds of these instabilities, which can be significantly lowered by the suprathermals. Suggestive examples are shown in Figure 9, in a diagram of proton anisotropy $\left(A_{p}\right)$ vs. proton (parallel) beta parameter $\left(\beta_{p \|}\right)$, not only by the thresholds of

\section{REFERENCES}

Anderson, B. J., Fuselier, S. A., and Murr, D. (1991). Electromagnetic Ion Cyclotron Waves Observed in the Plasma Depletion Layer. Geophys. Res. Lett. 18, 1955-1958. doi:10.1029/91GL02238

Bale, S. D., Kasper, J. C., Howes, G. G., Quataert, E., Salem, C., and Sundkvist, D. (2009). Magnetic Fluctuation Power Near Proton Temperature Anisotropy Instability Thresholds in the Solar Wind. Phys. Rev. Lett. 103, 211101. doi:10.1103/PhysRevLett.103.211101 proton firehose $(\mathrm{PFH})$ instability $\left(A_{p}<1\right)$, but also by the thresholds of EM ion-cyclotron (EMIC) instability in the upper part $\left(A_{p}>1\right)$ of this diagram (Shaaban et al., 2021a; Shaaban et al., 2021b). The anisotropy thresholds of electron instabilities are not shown here, but undergo the same influence in the presence of suprathermal electrons (Lazar et al., 2017b; Shaaban et al., 2019a; Lazar et al., 2019). This is another consequence of the presence of suprathermal populations, which can indirectly contribute to the accumulation of quasistable states in between these thresholds, near and along the isotropy condition $A \simeq 1$, as shown by the observations, not only for protons (Kasper et al., 2002; Bale et al., 2009) but also for electrons (Štverák et al., 2008). Other instabilities induced by beams or drifting populations (Verscharen et al., 2019; López et al., 2020; Micera et al., 2020; Schroeder et al., 2021), may show less systematic behavior when modeled by Kappa distributions (Shaaban et al., 2018a; Shaaban et al., 2020). Present resultss and recent advances in modeling the dispersion and stability of these anisotropic populations (López et al., 2021) should motivate future investigations to decode even more complex spectra of wave instabilities, as triggered by the interplay of various kinetic anisotropies of solar wind plasma populations, e.g., temperature anisotropy and relative drifts (Shaaban et al., 2018b; Shaaban and Lazar, 2020; Vasko et al., 2020), but also density gradients in phase space (Page et al., 2021).

\section{AUTHOR CONTRIBUTIONS}

All authors listed have made a substantial, direct, and intellectual contribution to the work and approved it for publication.

\section{FUNDING}

The authors acknowledge support from the Katholieke Universiteit Leuven, Ruhr-University Bochum, and ChristianAlbrechts University Kiel. SMS acknowledges the Alexandervon-Humboldt Research Fellowship. RAL acknowledges the support of ANID Chile through FONDECyT grant No. 11201048. These results were also obtained in the framework of the projects C14/19/089 (C1 project Internal Funds KU Leuven), G.0D07.19N (FWO-Vlaanderen), SIDC Data Exploitation (ESA Prodex-12), and Belspo project B2/191/P1/ SWiM. PHY acknowledges NASA Grant NNH18ZDA001N-HSR and NSF Grant 1842643 to the University of Maryland.

Camporeale, E., and Burgess, D. (2008). Electron Firehose Instability: Kinetic Linear Theory and Two-Dimensional Particle-In-Cell Simulations. J. Geophys. Res. 113, a-n. doi:10.1029/2008JA013043

Chew, G. F., Goldberger, M. L., and Low, F. E. (1956). The Boltzmann Equation an $\mathrm{D}$ the One-Fluid Hydromagnetic Equations in the Absence of Particle Collisions. Proc. R. Soc. Lond. A. 236, 112-118. doi:10.1098/rspa.1956.0116

Collier, M. R., Hamilton, D. C., Gloeckler, G., Bochsler, P., and Sheldon, R. B. (1996). Neon-20, Oxygen-16, and Helium-4 Densities, Temperatures, and Suprathermal Tails in the Solar Wind Determined with Wind/mass. Geophys. Res. Lett. 23, 1191-1194. doi:10.1029/96GL00621 
Coroniti, F. V., Kennel, C. F., Scarf, F. L., and Smith, E. J. (1982). Whistler Mode Turbulence in the Disturbed Solar Wind. J. Geophys. Res. 87, 6029-6044. doi:10.1029/JA087iA08p06029

Gary, S. P. (1993). Theory of Space Plasma Microinstabilities. Cambridge University Press. doi:10.1017/CBO9780511551512

Hau, L.-N., Fu, W.-Z., and Chuang, S.-H. (2009). Response to "Comment on 'Mathematical and Physical Aspects of Kappa Velocity Distribution' " [Phys. Plasmas 16, 094701 (2009)]. Phys. Plasmasphysics Plasmas 1616, 094701094702. doi:10.1063/1.3213389

He, J., Marsch, E., Tu, C., Yao, S., and Tian, H. (2011). Possible Evidence of AlfvénCyclotron Waves in the Angle Distribution of Magnetic Helicity of Solar Wind Turbulence. ApJ 731, 85. doi:10.1088/0004-637x/731/2/85

Hellberg, M. A., Mace, R. L., Baluku, T. K., Kourakis, I., and Saini, N. S. (2009). Comment on "Mathematical and Physical Aspects of Kappa Velocity Distribution" [Phys. Plasmas 14, 110702 (2007)]. Phys. Plasmas 16, 110702094701. doi:10.1063/1.3213388

Hellinger, P., Trávníček, P., Kasper, J. C., and Lazarus, A. J. (2006). Solar Wind Proton Temperature Anisotropy: Linear Theory and Wind/swe Observations. Geophys. Res. Lett. 33, L09101. doi:10.1029/2006GL025925

Hunana, P., and Zank, G. P. (2017). On the Parallel and Oblique Firehose Instability in Fluid Models. ApJ 839, 13. doi:10.3847/1538-4357/aa64e3

Jian, L. K., Russell, C. T., Luhmann, J. G., Strangeway, R. J., Leisner, J. S., and Galvin, A. B. (2009). Ion cyclotron waves in the solar wind observed by stereo near 1 au. ApJ 701, L105-L109. doi:10.1088/0004-637X/701/2/L105

Kasper, J. C., Lazarus, A. J., and Gary, S. P. (2002). Wind/swe Observations of Firehose Constraint on Solar Wind Proton Temperature Anisotropy. Geophys. Res. Lett. 29, 20-21. doi:10.1029/2002GL015128

Klein, K. G., Alterman, B. L., Stevens, M. L., Vech, D., and Kasper, J. C. (2018). Majority of Solar Wind Intervals Support Ion-Driven Instabilities. Phys. Rev. Lett. 120, 205102. doi:10.1103/PhysRevLett.120.205102

Lacombe, C., Alexandrova, O., Matteini, L., Santolík, O., Cornilleau-Wehrlin, N., Mangeney, A., et al. (2014). Whistler Mode Waves and the Electron Heat Flux in the Solar Wind:clusterobservations. ApJ 796, 5. doi:10.1088/0004-637x/796/1/5

Lazar, M., Fichtner, H., and Yoon, P. H. (2016). On the Interpretation and Applicability Ofk-Distributions. A\&A 589, A39. doi:10.1051/0004-6361/ 201527593

Lazar, M., López, R. A., Shaaban, S. M., Poedts, S., and Fichtner, H. (2019). Whistler Instability Stimulated by the Suprathermal Electrons Present in Space Plasmas. Astrophys. Space Sci. 364, 171. doi:10.1007/s10509-019-3661-6

Lazar, M., Pierrard, V., Poedts, S., and Fichtner, H. (2020). Characteristics of Solar Wind Suprathermal Halo Electrons. A\&A 642, A130. doi:10.1051/0004-6361/ 202038830

Lazar, M., Pierrard, V., Shaaban, S. M., Fichtner, H., and Poedts, S. (2017). Dual Maxwellian-Kappa Modeling of the Solar Wind Electrons: New Clues on the Temperature of Kappa Populations. A\&A 602, A44. doi:10.1051/0004-6361/ 201630194

Lazar, M., Poedts, S., and Fichtner, H. (2015). Destabilizing Effects of the Suprathermal Populations in the Solar Wind. A\&A 582, A124. doi:10.1051/ 0004-6361/201526509

Lazar, M., and Poedts, S. (2009). Firehose Instability in Space Plasmas with Bikappa Distributions. A\&A 494, 311-315. doi:10.1051/0004-6361:200811109

Lazar, M., Poedts, S., and Michno, M. J. (2013). Electromagnetic Electron WhistlerCyclotron Instability in Bi-kappa Distributed Plasmas. A\&A 554, A64. doi:10.1051/0004-6361/201220550

Lazar, M., Poedts, S., and Schlickeiser, R. (2011). Proton Firehose Instability in Bikappa Distributed Plasmas. A\&A 534, A116. doi:10.1051/0004-6361/201116982

Lazar, M., Poedts, S., and Schlickeiser, R. (2014). The Interplay of Kappa and Core Populations in the Solar Wind: Electromagnetic Electron Cyclotron Instability. J. Geophys. Res. Space Phys. 119, 9395-9406. doi:10.1002/2014JA020668

Lazar, M., Shaaban, S. M., Fichtner, H., and Poedts, S. (2018). Temperature Anisotropy Instabilities Stimulated by the Interplay of the Core and Halo Electrons in Space Plasmas. Phys. Plasmas 25, 022902. doi:10.1063/1.5016261

Lazar, M., Shaaban, S. M., Poedts, S., and Štverák, Š. (2017). Firehose Constraints of the Bi-kappa-distributed Electrons: a Zero-Order Approach for the Suprathermal Electrons in the Solar Wind. Mon. Not. R. Astron. Soc. 464, 564-571. doi:10.1093/mnras/stw2336

Lazar, M. (2012). The Electromagnetic Ion-Cyclotron Instability in Bi-kappa Distributed Plasmas. A\&A 547, A94. doi:10.1051/0004-6361/201219861
Lazar, M., Yoon, P. H., López, R. A., and Moya, P. S. (2018). Electromagnetic Electron Cyclotron Instability in the Solar Wind. J. Geophys. Res. Space Phys. 123, 6-19. doi:10.1002/2017JA024759

Li, X., and Habbal, S. R. (2000). Electron Kinetic Firehose Instability. J. Geophys. Res. 105, 27377-27385. doi:10.1029/2000JA000063

Lin, N., Kellogg, P. J., MacDowall, R. J., Scime, E. E., Balogh, A., Forsyth, R. J., et al. (1998). Very Low Frequency Waves in the Heliosphere: Ulysses Observations. J. Geophys. Res. 103, 12023-12035. doi:10.1029/98JA00764

Lion, S., Alexandrova, O., and Zaslavsky, A. (2016). COHERENT EVENTS AND SPECTRAL SHAPE AT ION KINETIC SCALES IN THE FAST SOLAR WIND TURBULENCE. ApJ 824, 47. doi:10.3847/0004-637x/824/1/47

Livadiotis, G., and McComas, D. J. (2012). Non-equilibrium Thermodynamic Processes: Space Plasmas and the Inner Heliosheath. ApJ 749, 11. doi:10.1088/ 0004-637x/749/1/11

López, R. A., Lazar, M., Shaaban, S. M., Poedts, S., and Moya, P. S. (2020). Alternative High-Plasma Beta Regimes of Electron Heat-Flux Instabilities in the Solar Wind. ApJ 900, L25. doi:10.3847/2041-8213/abaf56

López, R. A., Lazar, M., Shaaban, S. M., Poedts, S., Yoon, P. H., Viñas, A. F., et al. (2019). Particle-in-cell Simulations of Firehose Instability Driven by Bi-kappa Electrons. ApJ 873, L20. doi:10.3847/2041-8213/ab0c95

López, R. A., Shaaban, S. M., and Lazar, M. (2021). General Dispersion Properties of Magnetized Plasmas with Drifting Bi-kappa Distributions. DIS-K: Dispersion Solver for Kappa Plasmas. J. Plasma Phys. 87, 905870310. doi:10.1017/S0022377821000593

M. Lazar, R. Schlickeiser, S. Poedts, and M. Lazar (Editors) (2012). Exploring the Solar Wind (Rijeka: Intechopen Publishing), 241. doi:10.5772/2079

Mace, R. L., and Sydora, R. D. (2010). Parallel Whistler Instability in a Plasma with an Anisotropic Bi-kappa Distribution. J. Geophys. Res. 115, A07206. doi:10.1029/2009JA015064

Maksimovic, M., Gary, S. P., and Skoug, R. M. (2000). Solar Wind Electron Suprathermal Strength and Temperature Gradients: Ulysses Observations. J. Geophys. Res. 105, 18337-18350. doi:10.1029/2000JA900039

Maksimovic, M., Zouganelis, I., Chaufray, J.-Y., Issautier, K., Scime, E. E., Littleton, J. E., et al. (2005). Radial Evolution of the Electron Distribution Functions in the Fast Solar Wind between 0.3 and 1.5 AU. J. Geophys. Res. 110, A09104. doi:10.1029/2005JA011119

Maneva, Y., Lazar, M., Viñas, A., and Poedts, S. (2016). Mixing the Solar Wind Proton and Electron Scales: Effects of Electron Temperature Anisotropy on the Oblique Proton Firehose Instability. ApJ 832, 64. doi:10.3847/0004-637x/832/1/64

Marsch, E. (2006). Kinetic Physics of the Solar Corona and Solar Wind. Living Rev. Solar Phys. 3, 1. doi:10.12942/lrsp-2006-1

Mason, G. M., and Gloeckler, G. (2012). Power Law Distributions of Suprathermal Ions in the Quiet Solar Wind. Space Sci. Rev. 172, 241-251. doi:10.1007/s11214010-9741-0

Matteini, L., Hellinger, P., Landi, S., Trávníček, P. M., and Velli, M. (2012). Ion Kinetics in the Solar Wind: Coupling Global Expansion to Local Microphysics. Space Sci. Rev. 172, 373-396. doi:10.1007/s11214-011-9774-Z

Matteini, L., Landi, S., Hellinger, P., Pantellini, F., Maksimovic, M., Velli, M., et al. (2007). Evolution of the solar wind proton temperature anisotropy from 0.3 to 2.5 au. Geophys. Res. Lett. 34, L20105. doi:10.1029/2007GL030920

Micera, A., Zhukov, A. N., López, R. A., Innocenti, M. E., Lazar, M., Boella, E., et al. (2020). Particle-in-cell Simulation of Whistler Heat-Flux Instabilities in the Solar Wind: Heat-Flux Regulation and Electron Halo Formation. ApJL 903, L23. doi:10.3847/2041-8213/abc0e8

Nguyen, S. T., Perez, J. D., and Fennell, J. F. (2007). A Study of the Electromagnetic Proton Cyclotron Instability as a Generation Mechanism for EMIC Waves in the Earth's Magnetosphere Using SCATHA Data. J. Geophys. Res. 112, a-n. doi:10.1029/2007JA012291

Olbert, S. (1968). "Summary of Experimental Results from m.i.T. Detector on Imp1,". Physics of the Magnetosphere. Editors R. L. Carovillano, J. F. McClay, and H. R. Radoski (Springer Netherlands), 10, 641-659. doi:10.1007/978-94-0103467-8_23

Page, B., Vasko, I. Y., Artemyev, A. V., and Bale, S. D. (2021). Generation of HighFrequency Whistler Waves in the Earth's Quasi-Perpendicular Bow Shock. ApJL 919, L17. doi:10.3847/2041-8213/ac2748

Pierrard, V., and Lazar, M. (2010). Kappa Distributions: Theory and Applications in Space Plasmas. Sol. Phys. 267, 153-174. doi:10.1007/ s1 1207-010-9640-2 
Pierrard, V., Lazar, M., Poedts, S., Štverák, Š., Maksimovic, M., and Trávníček, P. M. (2016). The Electron Temperature and Anisotropy in the Solar Wind. Comparison of the Core and Halo Populations. Sol. Phys. 291, 2165-2179. doi:10.1007/s11207-016-0961-7

Podesta, J. J., and Gary, S. P. (2011). Magnetic Helicity Spectrum Of Solar Wind Fluctuations As A Function Of The Angle With Respect To The Local Mean Magnetic Field. ApJ 734, 15. doi:10.1088/0004-637x/734/1/15

Roberts, O. W., Alexandrova, O., Kajdič, P., Turc, L., Perrone, D., Escoubet, C. P., et al. (2017). Variability of the Magnetic Field Power Spectrum in the Solar Wind at Electron Scales. ApJ 850, 120. doi:10.3847/1538-4357/aa93e5

Scherer, K., Husidic, E., Lazar, M., and Fichtner, H. (2020). Generalized Anisotropic K-cookbook: 2D Fitting of Ulysses Electron Data. MNRAS 501, 606-613. doi:10.1093/mnras/staa3641

Schroeder, J. M., Boldyrev, S., and Astfalk, P. (2021). Stability of Superthermal Strahl Electrons in the Solar Wind. Monthly Notices R. Astronomical Soc. 507, 1329-1336. doi:10.1093/mnras/stab2228

Shaaban, S. M., Lazar, M., López, R. A., Fichtner, H., and Poedts, S. (2019). Firehose Instabilities Triggered by the Solar Wind Suprathermal Electrons. MNRAS 483, 5642-5648. doi:10.1093/mnras/sty3377

Shaaban, S. M., Lazar, M., López, R. A., and Poedts, S. (2020). Electromagnetic IonIon Instabilities in Space Plasmas: Effects of Suprathermal Populations. ApJ 899, 20. doi:10.3847/1538-4357/ab9cal

Shaaban, S. M., Lazar, M., and Poedts, S. (2018). Clarifying the Solar Wind Heat Flux Instabilities. MNRAS 480, 310-319. doi:10.1093/mnras/sty1567

Shaaban, S. M., Lazar, M., Poedts, S., and Elhanbaly, A. (2017). Shaping the Solar Wind Temperature Anisotropy by the Interplay of Electron and Proton Instabilities. Astrophys. Space Sci. 362, 13. doi:10.1007/s10509-016-2994-7

Shaaban, S. M., Lazar, M., Poedts, S., and Elhanbaly, A. (2016). The Interplay of the Solar Wind Proton Core and Halo Populations: EMIC Instability. J. Geophys. Res. Space Phys. 121, 6031-6047. doi:10.1002/2016JA022587

Shaaban, S. M., Lazar, M., and Schlickeiser, R. (2021a). Electromagnetic Ion Cyclotron Instability Stimulated by the Suprathermal Ions in Space Plasmas: A Quasi-Linear Approach. Phys. Plasmas 28, 022103. doi:10.1063/5.0035798

Shaaban, S. M., and Lazar, M. (2020). Whistler Instabilities from the Interplay of Electron Anisotropies in Space Plasmas: a Quasi-Linear Approach. MNRAS 492, 3529-3539. doi:10.1093/mnras/stz3569

Shaaban, S. M., Lazar, M., Wimmer-Schweingruber, R. F., and Fichtner, H. (2021b). A New Low-Beta Regime for Unstable Proton Firehose Modes in Bi-kappa-distributed Plasmas. ApJ 918, 37. doi:10.3847/1538-4357/ac0f01

Shaaban, S. M., Lazar, M., Yoon, P. H., and Poedts, S. (2018). Beaming Electromagnetic (Or Heat-Flux) Instabilities from the Interplay with the Electron Temperature Anisotropies. Phys. Plasmas 25, 082105. doi:10.1063/ 1.5042481

Shaaban, S. M., Lazar, M., Yoon, P. H., and Poedts, S. (2019). Quasilinear Approach of the Cumulative Whistler Instability in Fast Solar Wind: Constraints of Electron Temperature Anisotropy. AઐA 627, A76. doi:10.1051/0004-6361/ 201935515

Stansby, D., Horbury, T. S., Chen, C. H. K., and Matteini, L. (2016). Experimental Determination of Whistler Wave Dispersion Relation in the Solar Wind. ApJ 829, L16. doi:10.3847/2041-8205/829/1/116

Štverák, Š., Trávníček, P., Maksimovic, M., Marsch, E., Fazakerley, A. N., and Scime, E. E. (2008). Electron Temperature Anisotropy Constraints in the Solar Wind. J. Geophys. Res. 113, a-n. doi:10.1029/2007JA012733

Summers, D., and Thorne, R. M. (1991). The Modified Plasma Dispersion Function. Phys. Fluids B: Plasma Phys. 3, 1835-1847. doi:10.1063/1.859653

Thorne, R. M., and Summers, D. (1991). Enhancement of Wave Growth for Warm Plasmas with a High-Energy Tail Distribution. J. Geophys. Res. 96, 217-223. doi:10.1029/90JA01629
Tong, Y., Vasko, I. Y., Artemyev, A. V., Bale, S. D., and Mozer, F. S. (2019). Statistical study of whistler waves in the solar wind at 1 au. ApJ 878, 41 . doi:10.3847/1538-4357/ab1f05

Tong, Y., Vasko, I. Y., Pulupa, M., Mozer, F. S., Bale, S. D., Artemyev, A. V., et al. (2019). Whistler Wave Generation by Halo Electrons in the Solar Wind. ApJ 870, L6. doi:10.3847/2041-8213/aaf734

Vasko, I. Y., Kuzichev, I. V., Artemyev, A. V., Bale, S. D., Bonnell, J. W., and Mozer, F. S. (2020). On Quasi-Parallel Whistler Waves in the Solar Wind. Phys. Plasmas 27, 082902. doi:10.1063/5.0003401

Vasyliunas, V. M. (1968). A Survey of Low-Energy Electrons in the Evening Sector of the Magnetosphere with Ogo 1 and Ogo 3. J. Geophys. Res. 73, 2839-2884. doi:10.1029/JA073i009p02839

Verscharen, D., Chandran, B. D. G., Jeong, S.-Y., Salem, C. S., Pulupa, M. P., and Bale, S. D. (2019). Self-induced Scattering of Strahl Electrons in the Solar Wind. ApJ 886, 136. doi:10.3847/1538-4357/ab4c30

Vinas, A. F., Gurgiolo, C., Nieves-Chinchilla, T., Gary, S. P., Goldstein, M. L., Maksimovic, M., et al. (2010). "Whistler Waves Driven by Anisotropic Strahl Velocity Distributions: Cluster Observations," in Twelfth International Solar Wind Conference. Editors M. Maksimovic, K. Issautier, N. Meyer-Vernet, M. Moncuquet, and F. Pantellini (American Institute of Physics Conference Series), 1216, 265-270. doi:10.1063/1.3395852

Viñas, A. F., Moya, P. S., Navarro, R. E., Valdivia, J. A., Araneda, J. A., and Muñoz, V. (2015). Electromagnetic Fluctuations of the Whistler-Cyclotron and Firehose Instabilities in a Maxwellian and Tsallis-kappa-like Plasma. J. Geophys. Res. Space Phys. 120, 3307-3317. doi:10.1002/2014JA020554

Wicks, R. T., Alexander, R. L., Stevens, M., III, L. B. W., Moya, P. S., Viñas, A., et al. (2016). A PROTON-CYCLOTRON WAVE STORM GENERATED BY UNSTABLE PROTON DISTRIBUTION FUNCTIONS IN THE SOLAR WIND. ApJ 819, 6. doi:10.3847/0004-637x/819/1/6

Wilson III, L. B., Chen, L.-J., Wang, S., Schwartz, S. J., Turner, D. L., Stevens, M. L., et al. (2019). Electron Energy Partition across Interplanetary Shocks. I. Methodology and Data Product. ApJS 243, 8. doi:10.3847/1538-4365/ab22bd

Wilson, L. B., Koval, A., Szabo, A., Breneman, A., Cattell, C. A., Goetz, K., et al. (2013). Electromagnetic Waves and Electron Anisotropies Downstream of Supercritical Interplanetary Shocks. J. Geophys. Res. Space Phys. 118, 5-16. doi:10.1029/2012JA018167

Yoon, P. H. (2017). Kinetic Instabilities in the Solar Wind Driven by Temperature Anisotropies. Rev. Mod. Plasma Phys. 1, 4. doi:10.1007/s41614-017-0006-1

Yoon, P. H., and Seough, J. (2012). Quasilinear Theory of Anisotropy-Beta Relation for Combined Mirror and Proton Cyclotron Instabilities. J. Geophys. Res. 117, a-n. doi:10.1029/2012JA017697

Conflict of Interest: The authors declare that the research was conducted in the absence of any commercial or financial relationships that could be construed as a potential conflict of interest.

Publisher's Note: All claims expressed in this article are solely those of the authors and do not necessarily represent those of their affiliated organizations, or those of the publisher, the editors and the reviewers. Any product that may be evaluated in this article, or claim that may be made by its manufacturer, is not guaranteed or endorsed by the publisher.

Copyright $\odot 2022$ Lazar, López, Shaaban, Poedts, Yoon and Fichtner. This is an open-access article distributed under the terms of the Creative Commons Attribution License (CC BY). The use, distribution or reproduction in other forums is permitted, provided the original author(s) and the copyright owner(s) are credited and that the original publication in this journal is cited, in accordance with accepted academic practice. No use, distribution or reproduction is permitted which does not comply with these terms. 